\title{
MILLOUX INEQUALITY OF MEROMORPHIC FUNCTION IN ANNULI
}

\section{ZHAOJUN WU AND YUXIAN CHEN}

Abstract. The purpose of this paper is to establish the Milloux inequality of meromorphic function in annuli. We obtain a general form of Milloux inequality of meromorphic function in annuli when the multiple values are considered.

Mathematics subject classification (2010): Primary 30D30; Secondary 30D35, 30G30, 46G20. Keywords and phrases: Milloux inequality, meromorphic function, doubly connected domain.

\section{REFERENCES}

[1] T. B. CAO, Z. S. DENG, On the uniqueness of meromorphic functions that share three or two finite sets on annuli, Proceedings Mathematical Sciences, 122 (2012), 203-220.

[2] T. B. CAO, H. X. YI, Uniqueness theorems of meromorphic functions shares sets IM on Annuli, Acta Math. Sinica (Chin. Ser.), 54 (2011), 623-632.

[3] T. B. CAO, H. X. YI, H. Y. XU, On the multiple values and uniqueness of meromorphic functions on annuli, Comput. Math. Appl., 58 (2009), 1457-1465.

[4] Y. X. Chen, Z. J. Wu, Exceptional values of meromorphic functions and of their derivatives on annuli, Ann. Polon. Math. 105 (2012), 154-165.

[5] A. FERNÀNDEZ, On the value distribution of meromorphic function in the punctured plane, Mathematychni Studii, 34 (2010), 136-144.

[6] A. Ya. Khrystiyanyn, A. A. Kondratyuk, On the Nevanlinna Theory for meromorphic functions on annuli, I, Mathematychni Studii, 23 (2005), 19-30.

[7] A. Ya. Khrystiyanyn, A. A. Kondratyuk, On the Nevanlinna Theory for meromorphic functions on annuli, II, Mathematychni Studii, 24 (2005), 57-68.

[8] A. A. Kondratyuk, I. Laine, Meromorphic functions in multiply connected domains, Fourier series methods in complex analysis (Mekrijärvi, 2005) Univ. Juensuu Dept. Ser. No. 10 (2006), 9111.

[9] L. YANG, Value distribution theory, Translated and revised from the 1982 Chinese original, SpringerVerlag, Berlin; Science Press Beijing, Beijing, 1993. 